\title{
The HEGRA Experiment Status and Recent Results
}

\author{
F.A. Aharonian ${ }^{\mathrm{a}}$ and G. Heinzelmann ${ }^{\mathrm{b}}$ for the HEGRA collaboration \\ ${ }^{a}$ Max-Planck-Institut für Kernphysik, P.O.BOX 103980, D-69029 Heidelberg, Germany \\ ${ }^{\text {b} U n i v e r s i t a ̈ t ~ H a m b u r g, ~ I I . ~ I n s t . ~ f u ̈ r ~ E x p . ~ P h y s i k, ~ L u r u p e r ~ C h a u s s e e ~ 149, ~ D-22761 ~ H a m b u r g, ~ G e r m a n y ~}$
}

A report is given on the status and recent results from the HEGRA imaging atmospheric Cherenkov telescopes (IACT) concerning Mrk 501 and GRS 1915+105 and on recent results from the HEGRA arrays concerning galactic and extragalactic $\gamma$-sources, counterparts of TeV Gamma-Ray-Bursts, cosmic ray anisotropies, energy spectrum and composition of primary cosmic rays. The report summarizes the presentations given at the $15^{\text {th }}$ European Cosmic Ray Symposium 1996.

\section{GENERAL}

The HEGRA (High Energy Gamma Ray Astronomy) experiment is located at the Observatorio del Roque de los Muchachos on the Canary Island of La Palma, (2200 m a.s.l., $\left.28.75^{0} \mathrm{~N}, 17.89^{0} \mathrm{~W}\right)$ covering an area of about $200 \mathrm{~m} \cdot 200 \mathrm{~m}$. The layout is shown in Fig.1. The experiment consists of 6 imaging air Cherenkov telescopes and 3 arrays, the scintillator array, the wide angle Cherenkov array AIROBICC [1] and the Geiger tower array[2].

One of the major aims of the HEGRA experiment is the search for galactic and extragalactic sources of $\mathrm{TeV} \gamma$-radiation and a detailed study of their characteristics with the imaging atmospheric Cherenkov technique at energies above $500 \mathrm{GeV}$. Hereby the stereoscopic observation with the system of telescopes will be exploited. The $\mathrm{TeV} \gamma$-astronomy is continued towards higher energies $(\geq 20 \mathrm{TeV})$ with the three arrays. For the arrays a further important aim is a measurement of the energy spectrum and elemental composition of primary cosmic rays in the energy region from $\approx 500 \mathrm{TeV}$ to $\approx 10 \mathrm{PeV}$.

In the following first the status and performance of the imaging atmospheric Cherenkov telescopes and their recent results concerning Mrk 501 and the microquasar GRS1915+105 are given, then the recent results from the arrays are

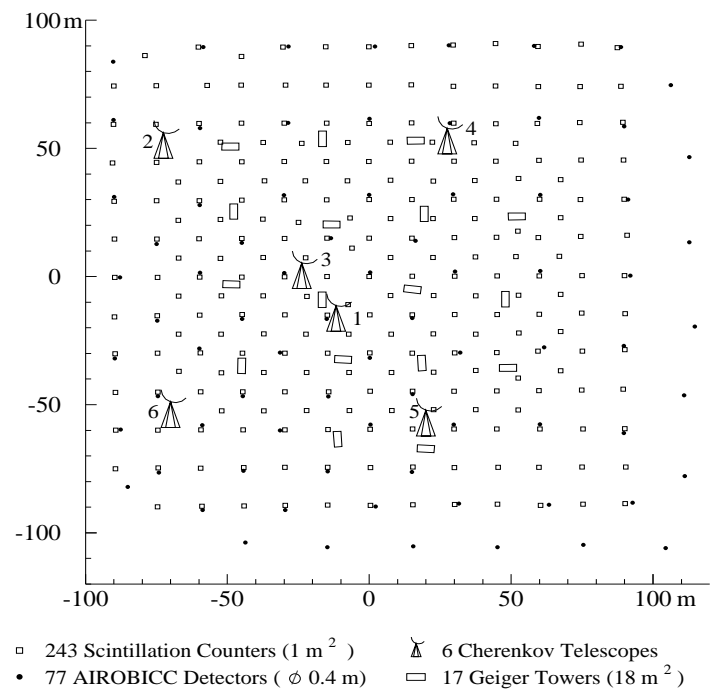

Figure 1. Layout of the HEGRA experiment

presented concerning galactic and extragalactic $\gamma$ sources, TeV counterparts of GRB's, cosmic ray anisotropies and the energy spectrum and elemental composition of the primary cosmic rays. 


\section{THE HEGRA SYSTEM OF IACTS}

\subsection{Telescopes}

Presently we are very close to complete a stereoscopic system consisting of 5 imaging telescopes (reported by G.Hermann and M.Ulrich at this symposium) which during the next several years will serve as one of the most powerful instruments in the field, operating at energies above $500 \mathrm{GeV}$ [3].

The multi mirror reflector of each telescope (Fig. 2) has an area $S_{\mathrm{mir}} \simeq 8.5 \mathrm{~m}^{2}$. The $60 \mathrm{~cm}$ diameter front aluminized and quartz coated spherical glass mirrors with a focal length of about $5 \mathrm{~m}$ are independently mounted on an almost spherical frame of an alt-azimuth mount. The angular resolution of the reflector is better than 10 arcminutes. The mount is driven by two stepping motors, and controlled by shaft encoders which provide a tracking accuracy of $0.02^{\circ}$.

Each telescope is equipped with a 271-channel camera [1] consisting of a close-packed cluster of circular PMTs (EMI 9083 KAFL) faced with hollow hexagonal-to-round light guides (Fig 3 ). The diameter of a pixel is about $0.24^{\circ}$, which results in the telescope's field of view $\mathrm{FoV} \approx 4.6^{\circ}$. The photon to photoelectron conversion efficiency including all kinds of losses (due to the mirror reflectivity, the quantum efficiency of PMTs, losses in light guides, etc.), and averaged over the spectrum of the Cherenkov light at the level of observations, is around $\eta_{\mathrm{ph} \rightarrow \mathrm{e}}=0.15$.

The entire electronics, including the trigger, readout, and the telescope control, is based on the VME standard. The digitization of the PMT pulses is performed with a Flash ADC system which allows to reconstruct the shape and time distribution of the pulses. The trigger decision is taken on two levels [5]. The fast trigger requires a minimum number of pixels to be fired, and the second (slower) level restricts the topology of an image. Near each telescope there is a small hut which houses the local electronics. Each telescope is equipped with a local CPU which communicates via an Ethernet line with the central computer. Although each telescope is built as stand

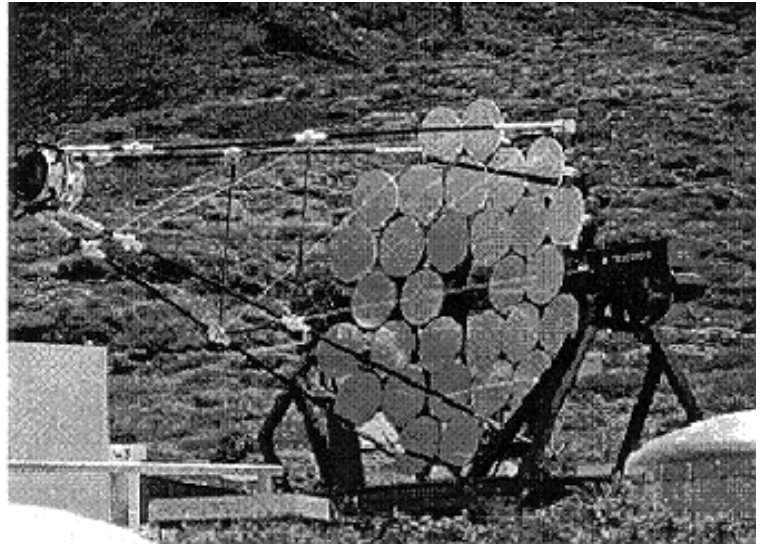

Figure 2. The HEGRA telescope CT-3.

alone detector, all telescopes can be integrated into the system by software command through the central computer (Pentium PC).

The first nominal 'system-telescope' called (for chronological reasons) CT-3, is in operation since August 1995. The data taken in the 95/96 season generally confirm the expected performance of a single telescope. The next 3 telescopes CT-4, CT-5, and CT-6 have been installed, tested and calibrated during July-December 1996, and are now operating together with CT-3 as a system in the 2-fold coincidence mode. The preliminary results are very encouraging and already demonstrate the power of the stereoscopic approach (see below). Currently, we use different FADC systems in different telescopes, but in the near future (until March 1997) we plan to operate the above four telescopes with the $120 \mathrm{MHz}$ FADC system. At approximately the same time we plan to replace the present 61 -channel (pixel size $0.43^{\circ}$, and $\mathrm{FoV} \simeq 3.9^{\circ}$ ) camera of CT-2 by a standard 271pixel high resolution camera. Thus we hope that after the test and calibration work, we will be able to run in the second half of 1997 the whole system as a very sensitive instrument for deep study of VHE $\gamma$-rays in the energy region $0.5-10 \mathrm{TeV}$.

In addition we plan to upgrade the first (prototype) HEGRA telescope CT-1. This equatorial mount telescope with $5 \mathrm{~m}^{2}$ reflector was in- 


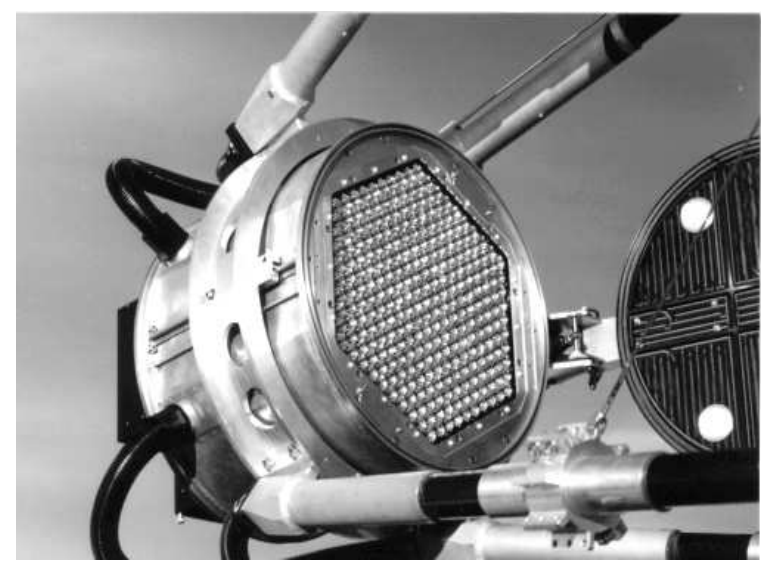

Figure 3. The 271-channel camera.

stalled and is taking data since 1992 [6]. In 1994 the original 37-channel camera was replaced by a new high resolution 127-channel camera (pixel size $0.24^{\circ}$ ) based on the same pixels used in the 'system' telescopes, but designed in the CAMAC standard [7]. In 1997 the round glass mirrors will be replaced by light hexagonal aluminum mirrors with a total reflector area $\simeq 12 \mathrm{~m}^{2}$. Thus the energy threshold of the telescope will be reduced to $500 \mathrm{GeV}$. We plan to use this telescope for individual observational programs, especially in immediate reaction to potential VHE $\gamma$-ray emitters showing flaring activity in other wavelengths. At the same time this telescope being located very near to the central telescope CT-3, can be effectively integrated, if necessary, with other IACTs in order to set up 2 or 3 independent stereoscopic IACT systems for simultaneous observations of different sources.

\subsection{Performance of the IACT system}

Although $\gamma$-ray showers of energy $500 \mathrm{GeV}$ with core position within $100 \mathrm{~m}$ from the telescope produce $\geq 100$ photoelectrons in the camera, sufficient for proper image analysis, the energy threshold of a single telescope is somewhat larger, and is determined by the suppression efficiency of the night sky background (N.S.B.) at the trigger level.

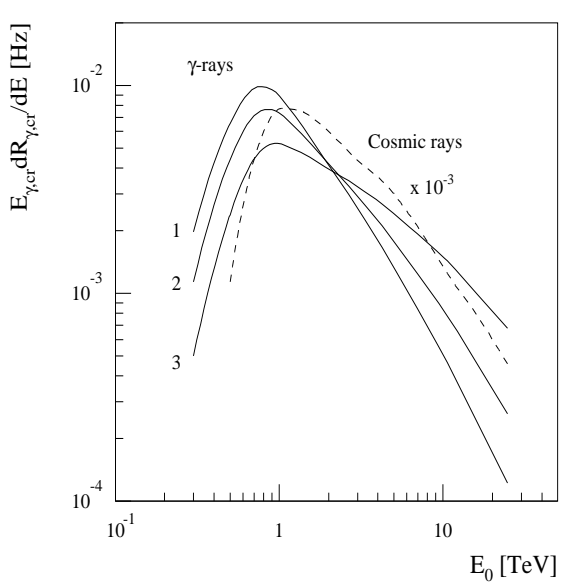

Figure 4. The hardware differential detection rates of cosmic rays and $\gamma$-rays by CT-3. The rates for $\gamma$ rayes are calculated assuming a power-law spectrum with spectral indices: (1) $-\alpha=3 ;(2)-\alpha=2.5$; (3) $\alpha=2$, and for an absolute flux of $10^{-11} \mathrm{ph} / \mathrm{cm}^{2} \mathrm{~s}$ above $1 \mathrm{TeV}$.

The hardware trigger criterion for each telescope, optimized by Monte Carlo calculations, is organized such that the signals in at least 2 neighboring pixels from the inner 169 pixels should exceed $\sim 15$ photoelectrons $(2 N N / 169 \geq 15)$. The Monte Carlo calculations of the detection rates of $\gamma$-rays from a point source, presented in Fig. 1. show that the effective energy threshold of the telescope CT-3 is between $700 \mathrm{GeV}$ and $1 \mathrm{TeV}$, depending on the slope of the primary photon spectrum. The first observations with CT-3 generally confirm the calculated performance of a single telescope, namely, a detection rate of $\gamma$-rays from the Crab Nebula above $700 \mathrm{GeV}$ of about $1.5 \times 10^{-3}$ photons/s on top of a cosmic ray $(\mathrm{CR})$ detection rate $\approx 5 \mathrm{~Hz}$, the significance of the signal per 1 hour of observations being $\simeq 2.2 \sigma$.

The requirement of simultaneous detection of showers by locally triggered $\geq 2$ telescopes significantly reduces the effect of the N.S.B. as well as that of the another important background connected with local muons. This allows a lower energy threshold of the system down to $\leq 500 \mathrm{GeV}$. 


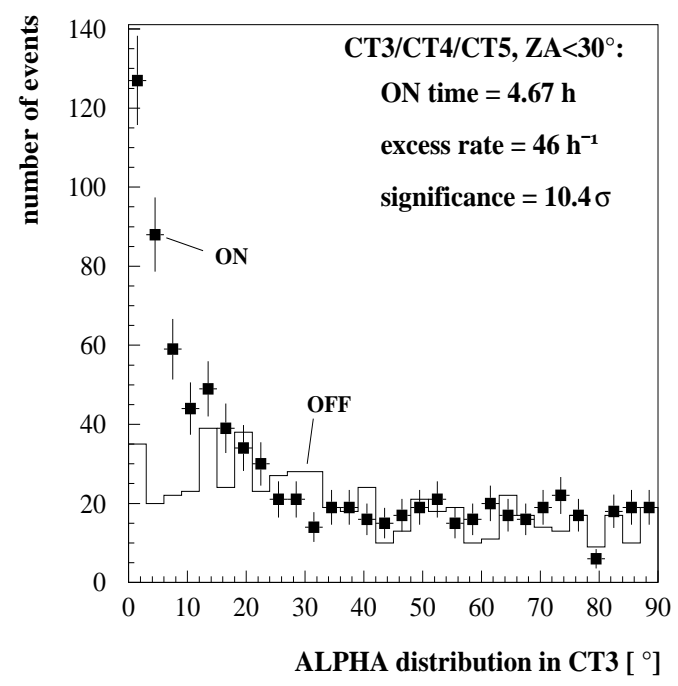

Figure 5. ALPHA-distribution for the Crab in the camera of CT-3 after the application of 'standard' image cuts in $\geq 2$ telescopes.

Also, the ability of the system to analyze the Cherenkov images in different projections where the images are only partially correlated, provides a superior rejection of hadronic showers. The preliminary results of observations of the Crab Nebula in October '96 by CT3/CT4/CT5, operating in the 2-fold telescope coincidence mode, generally confirm this prediction. In Fig. 5 we present the ON-source and OFF-source ALPHA distributions for the Crab in the camera of CT-3, obtained after filtering the events through the 'standard' image cuts of the showers detected by $\geq 2$ telescopes. This preliminary result shows that the significance of detection of $\simeq 500 \mathrm{GeV} \gamma$-rays from the Crab is achieved at a level of $\simeq 5 \sigma$ per 1 hour. In fact, we expect further significant improvement of the signal using energy and distance dependent 'cuts' to the detected images. (Note that the stereoscopic approach allows to determine the energy and the distance to the shower core with accuracies $\leq 20 \%$ and $\sim 10 \mathrm{~m}$, respectively). Furthermore, the obvious advantage of the joint operation of $\geq 2$ telescopes is the pos-

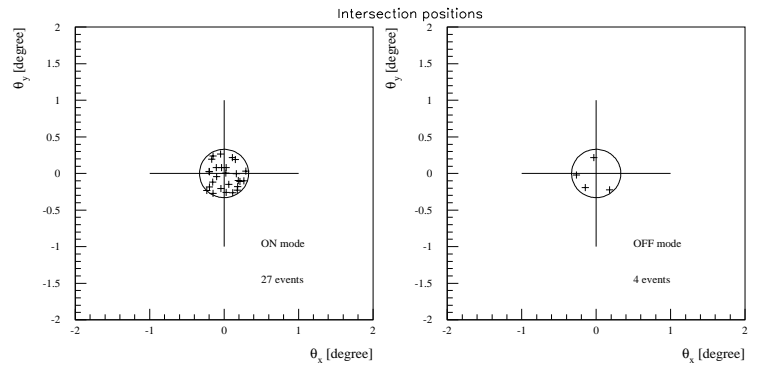

Figure 6. The reconstructed coordinates of the coincidence events observed by CT2 and CT3 within $0.3^{\circ}$ of the direction to the Crab (ON source events) and offset of the Crab (OFF source events) for 8.5 hour observations in both cases.

sibility to determine, without any a priory assumption, the arrival directions of individual $\gamma$ ray primaries with accuracy $\leq 0.1^{\circ}$. For the case of point-like sources, this provides by itself, rejection of the CR background based only on the orientational characteristics of showers by a factor of at least several 100. The procedure obviously requires good understanding of the characteristics of all telescopes linked in the system. Although the work regarding the CT3/CT4/CT5 data set is still in progress, our results obtained earlier using the combination of telescopes CT1/CT2 [8], and telescopes $\mathrm{CT} 2 / \mathrm{CT} 3$ allows us an optimistic view concerning the practical realization of this important aspect of the stereoscopic approach. Indeed, despite the short time of simultaneous observations from the Crab by CT- 2 and CT- 3 taken during the season 1995/1996 and a low coincidence rate (due to the high energy threshold of CT-2), the stereoscopic approach reveals a clear signal with 23 'excess' events against 4 (!) 'background' events within an angle $0.3^{\circ}$ around the direction to the Crab (see Fig. 6), the latter being in good agreement with the Monte Carlo predictions for the angular resolution of the CT2/CT3 system.

Thus, our current understanding of the system based on the recent experimental results as well as on comprehensive Monte Carlo studies, allows us to make conclusive predictions for the perfor- 
mance of the system of the 5 HEGRA imaging telescopes, namely

- energy threshold around $500 \mathrm{GeV}$;

- angular and energy resolutions of about $0.1^{\circ}$ and $\leq 20 \%$, respectively;

- minimum detectable fluxes of $\gamma$-rays $F_{\min } \sim$ $10^{-12} \mathrm{ph} / \mathrm{cm}^{2}$ s from point sources, and $F_{\min } \sim$ $10^{-11} \mathrm{ph} / \mathrm{cm}^{2} \mathrm{~s}$ from moderately extended sources with an angular size $\sim 1^{\circ}$.

\subsection{The recent results}

While the 5-IACT system is nearing the final stage of its commission, we continue to use the available telescopes in different configurations not only for methodological purposes, but also for observations of a number of astronomical objects. The first positive signal of $\gamma$-rays was seen from the Crab Nebula in 1992 by CT-1 in its first version [6]. Later we have detected $\mathrm{TeV} \gamma$-rays from the Crab with a significance of $\sim 10 \sigma$ using CT-2 [9]. The current sensitivity of the system consisting of CT3/CT4/CT5 has achieved a level which allows to obtain a strong signal from the Crab for just a few hours of observations under almost background-free conditions (see Fig. 5). Thus, we can now use the high energy photon beam of this persistent source for calibration purposes and for an understanding of detector characteristics.

Over the last several years CT-1 and CT-2 have been used for the search for $\mathrm{TeV} \gamma$-rays from other objects, in particular Cygnus X-3, AE Aquary, etc., reported earlier by different groups as possible episodic $\gamma$-ray emitters (see e.g. 10]). Unfortunately, we did not find DC $\gamma$-ray signals from any one of these sources. However, the observations of Markarian 421 in 1995 resulted in the independent detection of statistically significant positive signals by CT- 1 and CT-2 [11], thus confirming the discovery by the Whipple collaboration of $\mathrm{TeV}$ emission from this BL Lac object [12].

In 1996 we allocated an essential part of the time of the available telescopes for observations of relatively nearby AGNs, in particular Mrk 421 (CT1, CT2, CT3), Mrk 501, PKS 2209, MS 01166 (CT1), W Comae (CT1, CT2, and CT3, in the framework of the multiwavelength campaign),
Table 1

Summary of Mrk 501 and Crab observations with CT-1.

\begin{tabular}{lll}
\hline object & Mrk 501 & Crab \\
\hline ON-source obs. time $[\mathrm{h}]$ & 146.8 & 22.9 \\
ON events after all cuts & 1325 & 423 \\
expected background & 974 & 237 \\
number of excess events & 351 & 186 \\
excess rate $\left[\mathrm{h}^{-1}\right]$ & 2.4 & 8.1 \\
stat. significance (Li \& Ma) & 5.2 & 7.6 \\
\hline
\end{tabular}

and 1ES2344+514 (CT3/CT4/CT5). Also, CT2 and CT3 were used for observations of the superluminal galactic source GRS $1915+105$, and CT3/CT4/CT5 for observations of the shell type supernova remnant $\gamma$ Cygni. The analysis of most of these data is still in progress. Here we briefly discuss the results obtained from Mrk 501 and GRS $1915+105$.

\section{Mrk 501 (reported by S. Bradbury)}

This source is very similar to Mrk 421, and represents the same sub-population of AGNs called BL Lac objects - highly variable AGNs without strong emission lines, but showing a strong nonthermal component of radiation from radio to X-ray wavelengths. Recent EGRET observations showed that these objects are effective $\gamma$-ray emitters at energies $\geq 100 \mathrm{MeV}$ [13. Although Mrk 501 is not in the list of $\gamma$-ray emitting BL Lac's detected by EGRET, this source has been reported by the Whipple group to radiate in $\gamma$ rays above $300 \mathrm{GeV}$ at an average flux level of approximately $0.1 \mathrm{Crab}$ [14. The low flux of VHE $\gamma$-radiation from this source makes its detection rather difficult for our CT-1 and CT-2 telescopes. However, at the beginning of this year Mrk 501 started to show a flaring activity at VHE $\gamma$-rays (Weekes, private communication). This motivated us to allocate the CT-1 for long-term observations of Mrk 501. In order to obtain maximum exposure time the source was observed in tracking mode. Almost 150 hours of good quality data was obtained at zenith angles $\leq 25^{\circ}$ during 


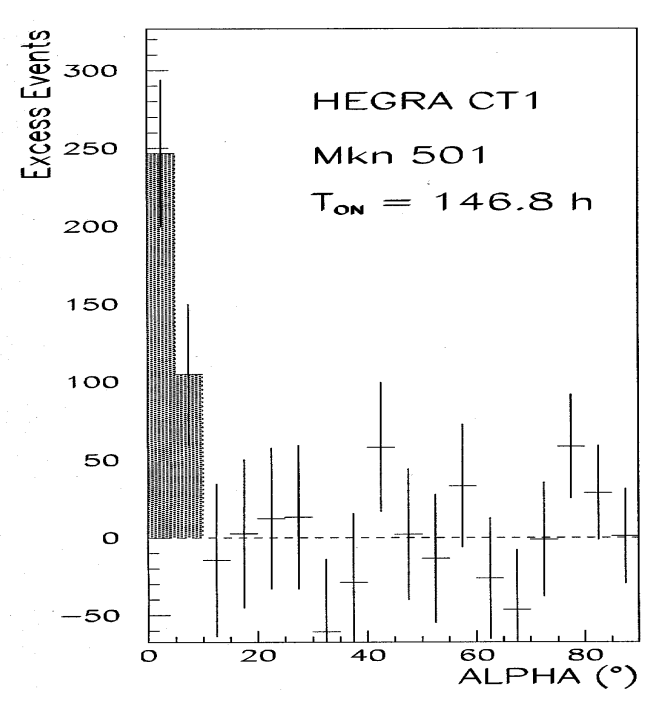

Figure 7. The ALPHA-distribution of the excess events for Mrk 501.

the period March-August 1996. The OFF-source observations required for background determination were made when Mrk 501 was not observable.

The ALPHA distribution for Mrk 501 shown in Fig. 7 indicates an excess of 351 events against expected $974 \pm 57$ background events (see Table 11) which can be interpreted as detection of $\gamma$-rays with a rate $R_{\gamma} \simeq 2.4 \mathrm{~h}^{-1}$ and statistical significance $5.2 \sigma$ [15]. Using the same procedure we find an excess rate of $\gamma$-like events from the direction of the Crab, $R_{\gamma} \simeq 8.1 \mathrm{~h}^{-1}$. This implies that during the observation period the flux from Mrk 501 was $\approx 3$ times lower than the Crab flux, i.e. approximately $2.5 \times 10^{-12} \mathrm{ph} / \mathrm{cm}^{2} \mathrm{~s}$. For comparison, the Whipple observations of Mrk 501 in 1995 showed lower flux relative to the Crab $(\approx 10 \%)$ at energies $\geq 300 \mathrm{GeV}$. This can be explained by different energy spectra of the Crab and Mrk 501 and/or by a strong variability of Mrk 501 which can be significant on timescales of 1 day [14]. The daily excess $\gamma$-ray event rates of our data are shown in Fig. 8. Above our threshold of $1.5 \mathrm{TeV}$ we have seen no significant "burst behaviour". Although the analysis of the distri-

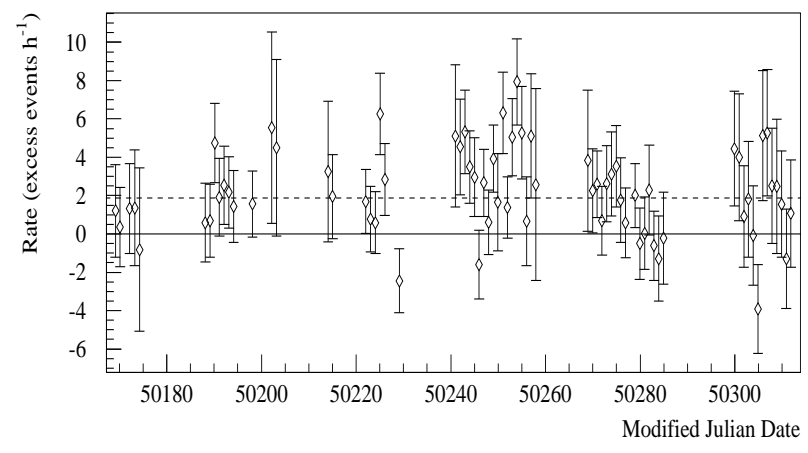

Figure 8. Daily excess $\gamma$-ray event rates from Mrk 501.

bution of total light content in the camera does not show a significant deviation of the spectral shape of Mrk 501 from the Crab spectrum, due to the uncertainty in the estimated power-law index $2.6 \pm 0.5$ we cannot make strong statements about modifications to the power law spectrum such as cut offs.

\section{GRS 1915+105 (reported by F.A. Aharonian)}

The recent discovery of the galactic superluminal source GRS 1915+105 [16], the scaled down analog of the AGN jets which are recognized as high energy $\gamma$-ray emitters, motivated the HEGRA collaboration to put this "microquasar" into the "Targets of Opportunity" list.

The first observations of the source in Spring 1995 with the telescopes CT1 (13 h) and CT2 $(25 \mathrm{~h})$ in ON/OFF mode showed a small evidence for a signal with statistical significance $2.8 \sigma$ and $2.6 \sigma$, respectively. However, the observations by the same telescopes after Aug 15 with doubled exposition time did not reveal any indication of a signal. Since this might be a result of a highly sporadic behavior of GRS 1915+105, we decided to continue observations in 1996.

In 1996 GRS 1915+105 has been observed during the period May-July 1996 by CT2 and CT3.

CT2 with an energy threshold $\approx 1 \mathrm{TeV}$ had been successfully used earlier for observations of the Crab [9] and Mrk 421 [11]. The main 


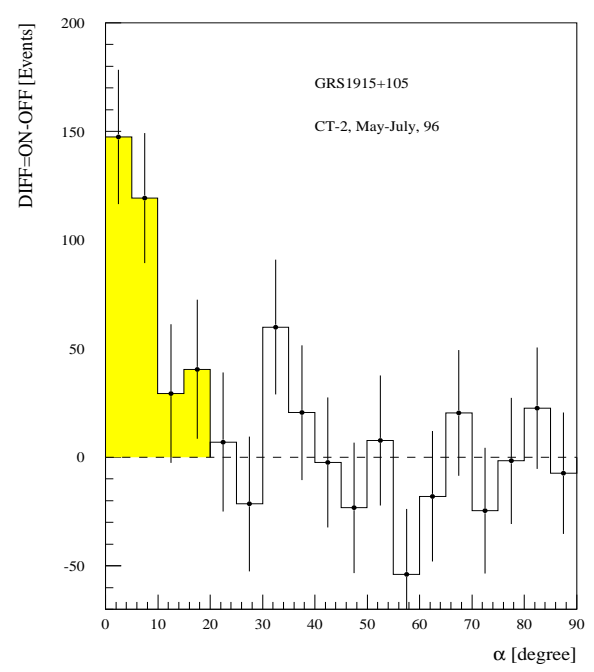

Figure 9. The ALPHA-distribution of the excess events for GRS 1915+105.

characteristics of this telescope are studied by Monte Carlo simulations which reproduce quite well the distributions of different image parameters. The rejection power of the telescope based on both shape and orientation parameters allows to suppress the CR background by a factor of 100 , the $\gamma$-ray acceptance being at the level of $\geq 50 \%$. The sensitivity of the telescope based on the observations of the Crab Nebula is estimated as $\approx 1.25 \sigma / \mathrm{h}$ detection rate of a signal from a point source at the flux level $J_{\gamma}(\geq$ $1 \mathrm{TeV}) \approx 10^{-11} \mathrm{ph} / \mathrm{cm}^{2} \mathrm{~s}$. Remarkably, this parameter has not been changed noticeably during the last 2 years which demonstrates the high stability of operation of the telescope.

Since GRS $1915+105$ is a highly variable object, we observed the source in the tracking mode. In addition, each month certain ON/OFF pairs were taken in order to monitor the shape of the background " $\alpha$-distribution". The statistics of observations at zenith angles $\theta \leq 30^{\circ}$ and the results are summarized in Table 2. The ALPHAdistribution of ON-OFF source events shown in Fig. 9 indicate the existence of a possible signal
Table 2

Summary of GRS $1915+105$ observations with CT-2.

\begin{tabular}{lcccc}
\hline & May & June & July & Total \\
\hline $\begin{array}{l}\text { ON-source obs. } \\
\text { time [h] }\end{array}$ & 9.89 & 37.12 & 23.83 & 70.84 \\
$\begin{array}{l}\text { ON events after } \\
\text { all cuts }\end{array}$ & 427 & 1527 & 944 & 2898 \\
$\begin{array}{l}\text { expected } \\
\text { background }\end{array}$ & 365 & 1373 & 875 & 2612 \\
$\begin{array}{l}\text { number of } \\
\text { excess events }\end{array}$ & 62 & 154 & 69 & 285 \\
$\begin{array}{l}\text { excess rate [h } \\
\text { stat. significance } \\
\text { (Li \& Ma) }\end{array}$ & 6.2 & 4.2 & 2.9 & 4.02 \\
\hline
\end{tabular}

with significance $4.7 \sigma$. The average rate of excess events is $\approx 4 \mathrm{~h}^{-1}$ which corresponds to $\approx 25 \%$ of the $\gamma$-ray detection from the Crab. Note that most of the "excess" events are due to the June period of observations which can be explained by $\approx 50 \%$ of statistics contained in this period. At the same time, perhaps one cannot exclude an intrinsic variability of the signal itself. For example the statistical significance of the signal in June 19 alone is of about $3 \sigma$, with a rate exceeding the average rate by a factor of 3 . This is seen in Fig. 10 where we present the daily rates of the "excess" events. Another feature of the time profile of the rate is its rather interesting coincidence with 3 prominent few-day-duration flares of GRS 1915+105 detected in radio and X-rays (see Fig. 10). This possible correlation in time of the rate of "excess" events may serve as an additional argument that strengthens the belief that the $\mathrm{TeV} \gamma$-ray detection from this source is a real effect rather than a result of statistical fluctuation of the background. If so, assuming that the radiation originates in the jet of GRS $1915+105$ approaching us, the intrinsic average TeV luminosity of the source is estimated as $10^{36} \mathrm{erg} / \mathrm{s}$.

However, we would like to emphasize that before going too far in the astrophysical interpretation of this statistically marginal signal, we obvi- 


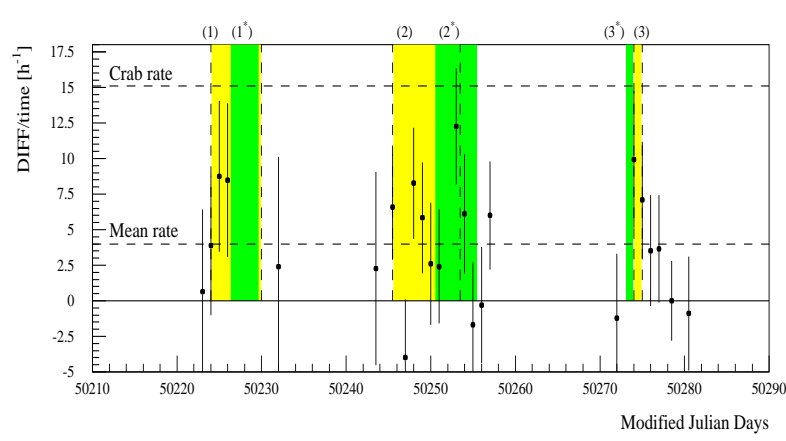

Figure 10. Daily rates of the excess events from GRS 1915+105. For comparison, the periods of large outbursts in radio and X-rays also are shown. The light hatched zones $(1,2,3)$ with borders indicated by dashed vertical lines corresponds to the periods of X-ray flares reported by Greiner J. et al., (Ap.J. Letters (in press); IAU Circ.6435), and the dark hatched zones $\left(1^{*}, 2^{*}, 3^{*}\right)$ correspond to the radio flares detected by the Ryle and Nancay telescopes (IAU Circ. $6411 ; 6432)$. A strong hard X-ray outburst in May 24-27 (MJD 50228-50231) has been reported also by the BATSE team (IAU Circ.6411). Note that at the time intervals without $\gamma$-ray data points correspond to the moonlight periods when the source could not be monitored.

ously need independent confirmations of this result by other instruments and groups. As was mentioned before, the source was observed simultaneously also by the CT-3 telescope. At the Perpignan Symposium, a positive signal was also shown from this telescope. Further analysis of these data however has revealed evidence for hardware problems during this observation period in some of the camera pixels, resulting in large spurious signals, which may fake $\gamma$-ray topologies. Work is in progress to correct these problems in software and to reprocess the data. At this point, no statements concerning GRS 1915+105 signals in CT-3 data can be made.

\section{RESULTS FROM THE ARRAYS}

\subsection{Angular resolution of AIROBICC}

In an analysis using Monte Carlo and experimental data it has been shown that the angular resolution of the AIROBICC array above a threshold of $18 \mathrm{TeV}$ for all events inside the array border is $\sigma_{63 \%}=0.29^{\circ}$. It decreases to $0.1^{\circ}$ at higher energies. The corresponding angular resolution of the scintillator array above $20 \mathrm{TeV}$ is $\sigma_{63 \%}=$ $0.9^{\circ}$ and reaches at higher energies $0.4^{\circ}$ [17]. The numbers given refer to hadron-initiated showers of vertical incidence. For photon-initiated showers an angular resolution of $\sigma_{63 \%}=0.22^{\circ}$ is expected according to Monte Carlo calculations for the AIROBICC array.

\subsection{Separation of gamma- and hadron- initiated showers with pearl}

A method to achieve a gamma-hadron separation has been presented by J. Prahl. The separation of gamma- and hadron-initiated showers is, besides a good angular resolution, crucial for a search of $\gamma$-ray sources.

The method called pearl uses first the well know fact that electromagnetic showers decline after the shower maximum faster than hadronic showers. Therefore the ratio $\mathrm{N}_{\mathrm{e}} / C_{L}$ of particles measured at ground $N_{e}$ and the number of Cherenkov photons $C_{L}$ is a well known separation quantity [18].

The other newly introduced quantity in the method pearl is related to the larger transverse extent of hadronic showers as compared to electromagnetic showers due to the hadronic transverse momentum arising in hadronic interactions. The quantity used for the transverse spread is defined by $r_{P}:=\sum_{i}\left\|\vec{r}_{i}-\vec{r}_{C}\right\| n_{i} / \sum_{i} n_{i}$ whereby $\vec{r}_{C}$ is the reconstructed core position, $\vec{r}_{i}$ the position of the $\mathrm{i}^{\text {th }}$ scintillator counter and $n_{i}$ the number of particles in the $\mathrm{i}^{\text {th }}$ scintillator counter. The separation quantity $r_{P}$ is related to the age parameter from a NKG fit.

Instead of using quantities obtained from a fit to the lateral distributions the method pearl uses the corresponding quantities derived from counting the amplitudes in the different counters i.e. 


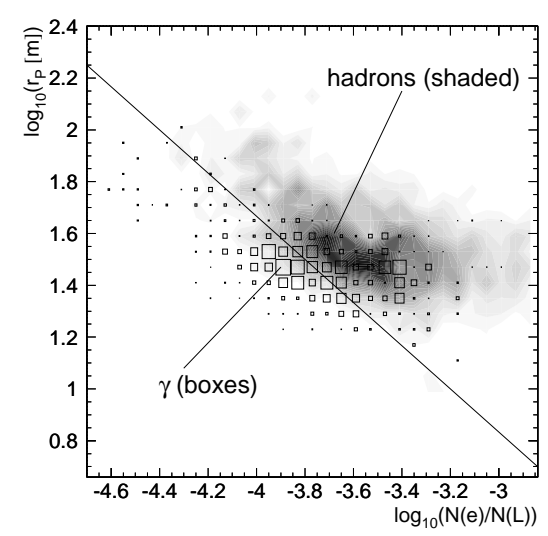

Figure 11. The separation quantities $r_{P}$ vs. $\mathrm{N}(\mathrm{e}) / \mathrm{N}(\mathrm{L})$ (s. text) for photon- and hadron-initiated showers (Monte Carlo). The straight line (pearl cut) indicated accepts $48 \%$ photon showers and rejects $90 \%$ hadron showers $(\mathrm{E} \geq 18 \mathrm{TeV})$.

the sum of the pulseheights in the scintillators $\mathrm{N}(\mathrm{e})$, the sum of the Cherenkovlight measured in the AIROBICC array $\mathrm{N}(\mathrm{L})$, and the quantity $r_{P}$ sensitive to the lateral extent defined above. The advantage of these quantities is that showers at the trigger threshold can be analysed without requiring a fit to converge. (Studies have shown that for showers for which the lateral fits converge the results are the same in the energy range of interest).

In Fig. 11 the two separation quantities are plotted against each other for photon and hadron initiated showers. A clear distinction can be seen and a straight line (pearl cut) can be used to separate photon- and hadron initiated shower in the 2-dim logarithmic plot. The $\mathrm{Q}$ factor defined as $Q:=\epsilon_{\gamma} / \sqrt{\epsilon_{\text {had }}}$ obtained for this method varies from $\mathrm{Q}=1.5$ at threshold of $18 \mathrm{TeV}$ to $\mathrm{Q}=2.5$ at $50 \mathrm{TeV}$.

\subsection{Galactic $\mathrm{TeV} \gamma$-ray sources}

With the gamma-hadron separation method pearl described above, based on the scintillator and AIROBICC arrays, a search for galactic sources has been performed using the 94/95 data. Amongst 13 selected sources the most significant excess of $3.3 \sigma$ after gamma-hadron separation is observed for Her X-1. No excess has

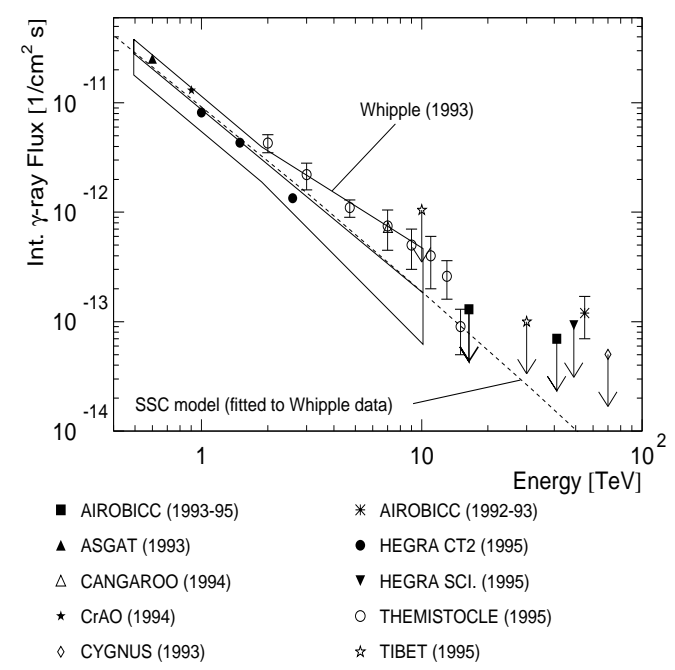

Figure 12. Integral $\gamma$-ray flux for the Crab nebula from the HEGRA Arrays (scintillator and AIROBICC) together with results from the HEGRA Cherenkov telescopes and other experiments.

been observed for the crab nebula in the $93 / 95$ data whereas in the 1992/93 data a $3.6 \sigma$ effect has been reported $\left(24^{t} h\right.$ ICRC Rom 1995, unpublished). The results for the Crab nebula are summarized in Fig. 12.

\subsection{Extragalactic $\mathrm{TeV} \gamma$-ray sources}

Up to now two extragalactic $\mathrm{TeV} \gamma$ sources Mrk 421 and Mrk 501 have been observed with the technique of imaging air Cherenkov telescopes (IACT) up to $\leq 10 \mathrm{TeV}$.

Whereas with the IACT only one source at a time can be observed, the arrays allow a simultaneous observation of several sources in the field of view of HEGRA, however for the prize of a higher energy threshold. This may severely limit the chance of an observation due to the absorption of $\mathrm{TeV}$ photons on the not well known infraredoptical background fields ( $\gamma$-ray horizon).

In order to perform a search a list of 11 source candidates with redshifts $\leq 0.1$ has been set up, belonging to the class of blazars visible in the field of view of HEGRA [19]. The sources have been 
looked at in the stacking mode and individually.

The search has been performed for datasets with and without gamma-hadron separation capability. The gamma-hadron separation with the GEIGER towers using a neural net analysis (Qfactors 2 - 2.5) 20] requires a threshold of 50 $\mathrm{TeV}$ and an angular bin of 1 degree (using the direction of the scintillator array) therefore the results from other data sets are for comparison also evaluated for $50 \mathrm{TeV}$ and 1 degree. Stacking all source candidates the highest significances reported by $\mathrm{N}$. Magnussen are observed in the scintillator data from 6.89 -6.92 without gammahadron separation $(3.5 \sigma)$ and from 11.94-3.95 with gamma-hadron separation using the Geiger towers $(4.0 \sigma)$. The most prominent single source candidate is $0116+319$ which contributes with $4.4 \sigma$ in the scintillator data. No excess has been observed for a sample of blazars with higher redshifts and for a sample of 49 galaxies characterized by steep radio spectra.

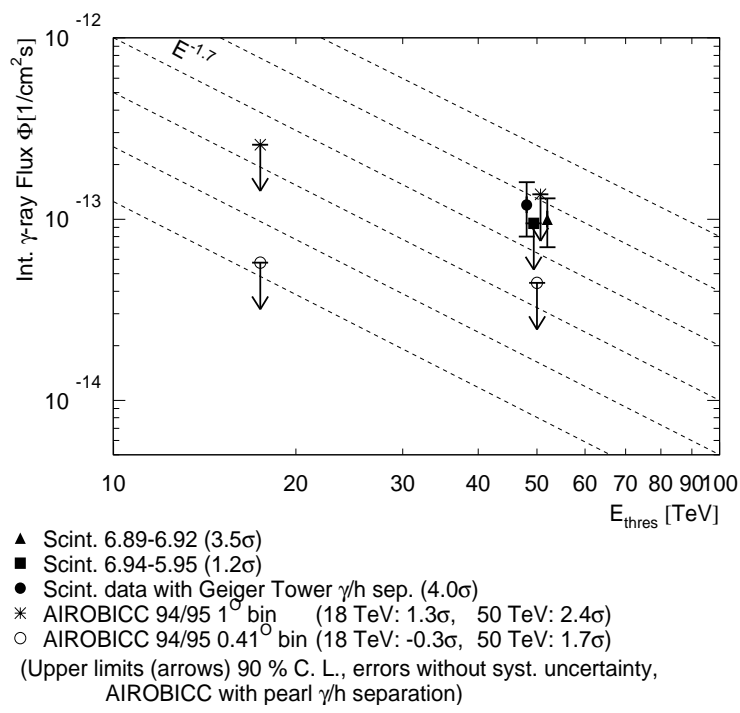

Figure 13. Comparison of the results from different analyses and data samples (s. text) on a flux scale for a generic blazar (from a superposition of 11 nearby blazars)
As reported by J. Prahl the scintillator data from 1994/95 show an excess of $1.2 \sigma$ and the AIROBICC data from 1994/95 with $\gamma$-hadron separation $2.4 \sigma$. Exploiting the much better angular resolution and lower energy thresholds possible for the AIROBICC data, the significances do not increase as expected but decrease. In order to compare the results of the different data sets the excesses with the highest significance are converted tentatively into a flux and the other results in upper limits (Fig. 13).

A definite conclusion cannot yet be drawn and more systematic checks are under way especially concerning the absolute pointing which is crucial in order to exploit the good angular resolution of the AIROBICC array. Also further AIROBICC data from 1992/93 are investigated. If a signal could be established this would imply, that the infrared background radiation has to be much less than presently assumed and the sources according to present day models would probably be proton accelerators.

\subsection{TeV counterparts of gamma-ray bursts}

Gamma-Ray-Bursts (GRB's) are known for more than 25 years yet their physical origin and distance scale are still unknown. Observations of $\mathrm{TeV}$ counterparts would impose limits on the unknown distance scale because of the absorption at the infrared intergalactic background fields which limits the mean free path of $\mathrm{TeV}$ Photons to distances $\leq 100 \mathrm{Mpc}$.

To search for $\mathrm{TeV}$ emission associated with gamma-ray bursts registered by the BATSE instrument all HEGRA detector components have been used. Especially searches with the large field of view of the air shower arrays have been performed for $\mathrm{TeV}$ emission which may occur simultaneous, preceding or following a burst. A delayed emission may be expected if GRB's account for the acceleration of protons up to the highest energies which in turn generate a secondary intergalactic electromagnetic cascade due to interactions with the microwave background fields.

No significant signal has been observed for the different searches as reported by B. Funk and H. Krawczynski. The results from a search for co- 


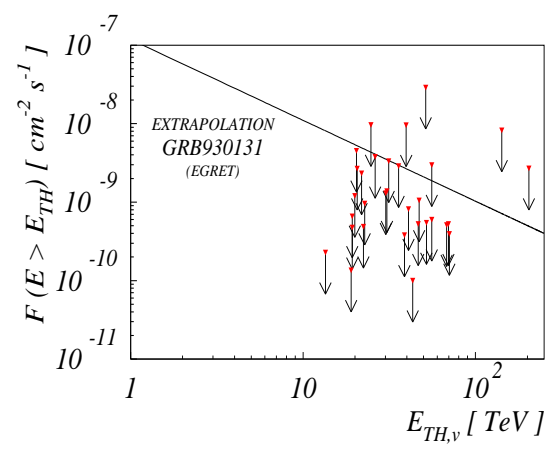

Figure 14. For 31 BATSE bursts (1994-1996) the HEGRA upper limits on the coincident integral flux (90\% Confidence Level) as a function of the threshold energy for inducing photons are shown. The threshold energy is a function of the zenith angle of the location of a burst.

incident emission obtained for 31 BATSE bursts are shown in Fig. 14. To guide the eye an extrapolated GRB spectrum [21] is displayed.

In future a lower energy threshold of about 2 $\mathrm{TeV}$ will be possible due to higher data taking rate and an angle sensitive trigger.

\subsection{Cosmic ray anisotropies}

A search for anisotropies has been reported by D. Schmele using the data taken during 1994/95 with the scintillator array (60 Mio events), with the AIROBICC array (50 Mio) and in the data with the AIROBICC array using the $\gamma$-hadron separation method pearl.

In order to search for anisotropies, all data (for each data set separately) have been used to determine a two dimensional shape of the background distribution in local coordinates $\phi$ and $\theta$. This shape of the background distribution is corrected for the air pressure by a multiplicative term depending on $\theta$ and air pressure. Using this (pressure corrected) background shape in local coordinates a background map in equatorial coordinates is generated according to the actual event rates recorded. In order to obtain the actual event rates in this procedure, the experimental event rates have first been corrected for the (variable) deadtime and air pressure. In this way the actual up

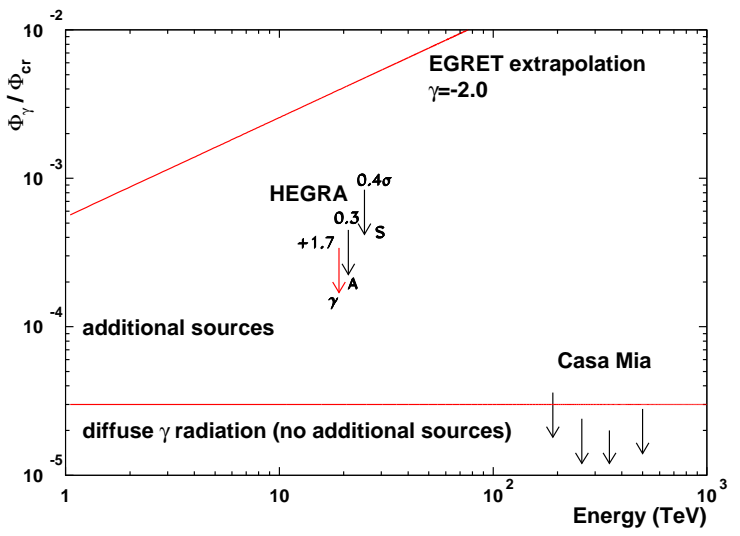

Figure 15. $\Phi_{\gamma} / \Phi_{C R}$ upper limit (90\% CL) from the galactic disc $(\mathrm{S}=$ scintillator, $\mathrm{A}=\mathrm{AIROBICC}, \gamma=$ with pearl $\gamma$-hadron separation). Numbers are Li-Ma significances.

time and performance of the array is taken into account.

The deviations of the data from the background (for bin sizes corresponding to the angular resolution) follow a normal distribution $\mathrm{N}(0,1)$ rather well. For binsizes from $1^{\circ}$ to $50^{\circ}$ an upper limit of $\Phi_{C R} / \Phi_{B G} \leq 10^{-2}$ for the smallest bin and $\leq 10^{-3}$ for the largest bin is obtained. These values are essentially determined by the statistics of $\sim 50$ Mio events per event sample.

In order to look for an emission from the galactic disk, the maps are transformed into galactic coordinates and projected onto galactic latitudes and compared. A disk of width $\|b\| \leq 10^{\circ}$ has been assumed $\left(1=30^{\circ}-220^{\circ}\right)$. The results for the upper limits for a flux from the galactic disk for the three data sets are shown in Fig.15. Also shown are the limits of CASA MIA [22], the expected theoretical flux of $3 \times 10^{-5}$ for diffuse emission from interaction of cosmic rays with interstellar matter $\|b\| \leq 5^{\circ}$ [23] and a naive EGRET extrapolation with a constant slope of $\gamma=2$. The HEGRA limits of $\Phi_{\gamma} / \Phi_{C R} \geq 4 \times 10^{-4}$ can be interpreted as limits for an additional flux due to 'unresolved' galactic sources in the energy range above $20 \mathrm{TeV}$.

Finally a search for a contribution from the region around the direction of the EHE events 
as seen by Fly's Eye and Yakutsk has been performed. A contribution in the $\mathrm{TeV}$ energy range may be expected via the intergalactic cascade $\left(p+\gamma_{3 K} \rightarrow X+\gamma_{T e V}\right)$. On the scale from $5^{\circ}$ to $20^{\circ}$ radius around the Fly's Eye event position $(\mathrm{RA}=85.2, \mathrm{DEC}=48.0)$ 24], no excess has been found. An upper limit for the contribution of an EHE source $\Phi_{\text {source }} / \Phi_{B G} \sim 10^{-3}$ above $20 \mathrm{TeV}$ can be given. This limit includes a possible contribution from the galactic disk, which is near to the estimated source position.

All searches are carried out with the three data samples described above and all three agree well within their different sensitivities and statistics. In the near future the size of the data sample will will be increased substantially.

\subsection{Primary energy and composition}

The measurement of the energy and composition of primary cosmic rays in the energy range of the knee is only accessible to the indirect methods of air showers and is still controversial and a matter of debate. Here a new method is presented to reconstruct the energy and masses of primary particles with energies above $300 \mathrm{TeV}$ from the electromagnetic component of an air shower as measured by a scintillator array and an array of Cherenkov counters. The main steps of the reconstruction procedure as presented by H. Krawczynski, can be summarized as follows:

(1) The distance to the shower maximum $D_{\max }$ is reconstructed from the lateral distribution of the Cherenkov light as measured at detection level. It can be shown by Monte Carlo simulation that $\mathrm{D}_{\max }$ can be determined independent of the primary mass (and almost independent of energy). This is due to the fact that the shower development behind the shower maximum, which dominates the determination of the distance to the maximum, is independent of the primary mass of nuclei.

(2) Electromagnetic energy $E_{\text {em }}$. From the number of electrons $N_{e}$ as obtained from a NKG fit and the distance to the showermaximum $D_{\max }$ the number of electrons in the maximum $N_{e, \max }$ can be inferred. This again is independent of the the particle mass. The number of electrons in
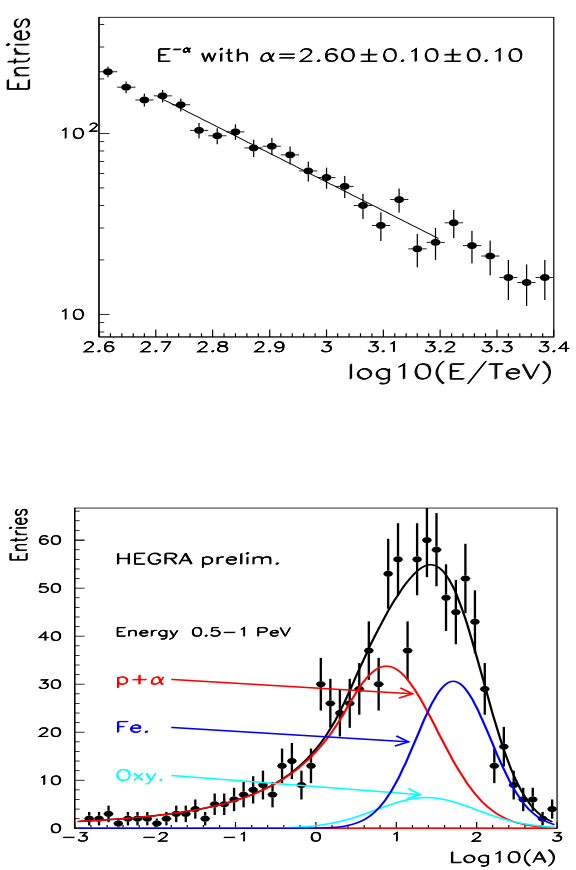

Figure 16. The upper part shows the distribution of the reconstructed energies. On the lower part the distribution of the reconstructed nucleon number of all events with reconstructed energies between $500 \mathrm{TeV}$ and $1 \mathrm{PeV}$ is shown. The abundances of three groups of primaries have been determined with a three component fit of the distributions predicted from Monte Carlo data for each group (dotted lines) to the measured distribution (solid line).

the maximum is in a good approximation proportional to the electromagnetic energy $E_{e m}$.

(3) Energy per nucleon $E / A$. From the distance to the showermaximum the penetration depth is obtained. Since the penetration depth depends (logarithmically) on E/A, the energy per nucleon E/A can be (coarsely) determined.

(4) Primary energy E. The fraction of the total energy which is transformed into electromagnetic energy increases in a known way (i.e. from Monte Carlo simulations) with the energy/nuleon: $\mathrm{E}_{e m}=\mathrm{f}(\mathrm{E} / \mathrm{A})$. With $\mathrm{E} / \mathrm{A}$ from step (3) and $\mathrm{E}_{\text {em }}$ from step (2) the total energy $\mathrm{E}$ can 
be determined.

(5) Primary nucleon number $A$. With E and E/A known from step (3) and (4) also the nucleon number A is coarsely known. More details of the method can be found in [26].

In order to first test the new method, it is applied to the energy range from $500 \mathrm{TeV}$ to $1 \mathrm{PeV}$ where the composition is known or can be extrapolated from direct measurements taken with satellites and balloone-borne experiment. The preliminary results shown in Fig. 16 are in good agreement with the results from direct measurements. For the differential energy spectrum a spectral index of $2.60 \pm 0.10 \pm 0.1$ is obtained in the energy range from $500 \mathrm{TeV}$ to $2 \mathrm{PeV}$. The abundance of light nuclei to all nuclei is obtained to be $0.62 \pm 0.05 \pm 0.10$ in the energy range from 500 $\mathrm{TeV}$ to $1 \mathrm{PeV}$. The distributions in Fig.16 reflect the fluctuations in the penetration depth for the different primaries. It should be stressed that for the mass determination the mean values as well as width (i.e. fluctuations) of the curves contribute.

Results with a slightly different method have been reported at $24^{\text {th }}$ ICRC Rom 27. In the near future the new method is applied to the energy range above $1 \mathrm{PeV}$ (knee).

\section{Acknowledgements}

The HEGRA Collaboration thanks the Instituto de Astrofisica de Canarias for the use of the HEGRA site at the Roque de los Muchachos and its facilities. This work was supported by the $\mathrm{BMBF}$, the DFG and the CICYT.

\section{REFERENCES}

1. A.Karle et al., Astroparticle Physics 3 (1995) 321

2. W. Rhode et al., NIM A378 (1996) 399

3. F.A.Aharonian et al., Proc. "Towards a Major Atmospheric Cherenkov Detector-II", Calgary (1993) 81.

4. M.Panter et al., Proc. 24th ICRC, Rome 1 (1995) 958.

5. G.Hermann, Proc. "Towards a Major Atmospheric Cherenkov Detector-IV", Padova (1995) 81.

6. R.Mirzoyan et al., NIM A351 (1994) 51.

7. G.Rauterberg et al., Proc. 24th ICRC, Rome 3 (1995) 460 .

8. A.Kohnle et al., Astroparticle Physics 5 (1966) 119

9. A. Konopelko et al., Astroparticle Physics 4 (1996) 199

10. T.C.Weekes, Space Sci. Rev. 59 (1992) 315.

11. D.Petry et al., Astron. Astrophys. 311 (1996) L13.

12. M.Punch et al., Nature 358 (1992) 477.

13. von Montigny C. et al., Ap.J. 440 (1996) 525.

14. J.Quinn et al., Ap.J. 456 (1996) L83.

15. S.Bradbury et al., Astron. Astrophys., submitted.

16. I.F.Mirabel and L.F.Rodriguez, Nature 371 (1994) 46.

17. H. Krawczynski et al., NIM A (1996) in press

18. F. Arqueros et al., 24th ICRC Rom

19. K. Mannheim et al., Astron. \& Astrophys. 315 (1996) 77

20. S. Westerhoff et al., Astroparticle Physics 4 (1995) 119.

21. M. Sommer et al., Astrophys. J. 422 (1994) L63

22. A. Borione et al., 24th ICRC Rom 2 (1995) 463

23. F.A. Aharonian and A.M. Atoyan, Astron. Astrophys 917A (1996) 309

24. Bird, D.J. et al., Astrophys. J. 441 (1995) 114

25. Nagano, M. and Takahara, F. ; Astrophysical Aspects of Most Energetic Cosmic Rays; World Scientific, Singapore; (1991)

26. A. Lindner astro-ph/9611175 publication in preparation

27. R. Plaga et al.,24th ICRC Rom 2 (1995) 693 